\title{
Un Programa de Capacitación Previo al Inicio de la Práctica Profesional de Médicos Peruanos Mejora sus Conocimientos sobre Atención de Parto y Partograma
}

\author{
A Training Program Prior to Professional \\ Practice by Peruvian Doctors Improves their \\ Knowledge on Childbirth and the Partograph
}

PALABRAS CLAVE:

- Partograma

- Parto

- Educación

KEYWORDS:

- Delivery

- Parturition

- Education.

Recebido em: 15/03/2010

Reencaminhado em: 16/08/2010

Aprovado em: 23/11/2010

REVISTA BRASILEIRA DE EDUCAÇC̄O MÉDICA $186 \overline{35(2): 186-122 ; 2011}$
Jorge Renato Aparicio Poncel Sergio Gerardo Ronceros Medrano

Karim Goebel Szielaskoll

Ericson Leonardo Gutierrez Ingunza

\section{RESUMEN:}

Se realizó un estudio analítico, longitudinal y prospectivo; se aplicó un cuestionario y lista de cotejo determinando el nivel de conocimientos y aplicación previos sobre el parto y el partograma a 72 internos de medicina que rotaron por el servicio de Gineco-Obstetricia del Hospital Nacional Dos de Mayo de Lima-Perú, entre enero a diciembre del 2006, posteriormente se aplicó el mismo instrumento a la mitad y al final del estudio. Para el análisis estadístico se utilizó la prueba de análisis de varianza (ANOVA), los cálculos se realizaron con un nivel de confianza de 95\%. Se encontró un aumento significativo en el nivel de conocimiento sobre el parto, el partograma y la aplicación en la elaboración del partograma $(p<0.001)$. La importancia de este estudio radica en que la mayoría de los médicos peruanos iniciarán su práctica profesional en servicios de atención primaria alejados de los centros de atención de mayor complejidad, donde tendrán que realizar la atención de parto. Así mismo este programa les ayudará a reconocer el momento indicado para realizar una referencia a un servicio de salud de mayor complejidad.

\section{ABSTRACT}

An analytical, longitudinal, and prospective study was performed, with the use of a questionnaire and checklist to determine the level of knowledge and prior application in deliveries and the partograph, with 72 medical interns that went through rotation at the Obstetrics and Gynecology Department of the "Dos de Mayo" National Hospital in Lima, Peru, from January to December 2006. The same instrument was later applied half way through and at the end of the study. Statistical analysis used analysis of variance (ANOVA), and the calculations were performed with a 95\% confidence interval. The results showed a significant increase in the level of knowledge on childbirth, the partograph, and application in the elaboration of the partograph $(p<0.001)$. The study's importance lies in the fact that the majority of Peruvian physicians begin their professional practice in primary care services (far from medical centers with higher complexity), where they will have to perform deliveries. The program will also help them recognize the right moment to transfer the patient to a health center with higher complexity.
${ }^{I}$ Universidad Nacional Mayor de San Marcos, Lima, Perú.

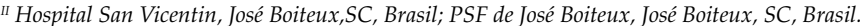




\section{INTRODUCCIÓN}

En nuestro país el proceso de enseñanza y aprendizaje en ciencias de la salud, en muchos casos, todavía usa el modelo pedagógico de la transmisión, es decir el expositivo, favoreciéndose la repetición, la falta de sentido crítico, la rutina en la docencia y la pasividad en el aprendizaje. Sin embargo, el proceso actual ha ido demostrando que la educación de adultos debe centrarse en el estudiante y los métodos tradicionales deben dejar el lugar a formas más productivas. Las estrategias de la educación basada en problemas han ido difundiéndose en forma rápida y esto se corrobora en múltiples publicaciones ${ }^{1}$, dándose actualmente un enfoque constructivista al proceso de enseñanza y aprendizaje. De esta manera, el sentido del profesor de antaño cambia al de facilitador o tutor ${ }^{2}$, contribuyendo al aprendizaje. En su rol de facilitar al estudiante la construcción de conocimientos, debe motivarlo, generando curiosidad y despertando la inquietud por descubrir ${ }^{3}$.

Está demostrado que el partograma puede ser una herramienta de bajo costo, efectiva para monitorizar el trabajo de parto y predecir sus complicaciones, sumamente importante sobre todo en países en desarrollo ${ }^{4}$.

A pesar de esto, en la práctica clínica se ha observado que en el Hospital Nacional "Dos de Mayo", una sede docente donde confluyen Internos de Medicina de diversas universidades del Perú, este instrumento no es empleado de forma correcta, tanto en su ejecución, como en su elaboración oportuna.

Por esta razón se realizó el presente estudio, cuyo objetivo general fue determinar los cambios en el nivel de conocimientos para la elaboración del partograma con curva de alerta por los Internos de Medicina del Hospital Nacional “Dos de Mayo" durante el año 2006, luego de recibir el programa de capacitación "Partograma con curva de alerta".

\section{MATERIALES Y MÉTODOS}

El estudio fue de tipo Analítico-Longitudinal-Prospectivo. Se realizó en el Departamento de Ginecología y Obstetricia del Hospital Nacional "Dos de Mayo“. Este es un hospital de tercer nivel, ubicado en el centro de Lima (Perú) y es uno de los más antiguos centros de formación de médicos peruanos. El estudio incluyó a todos los Internos de Medicina que rotaron por el Departamento de Ginecología y Obstetricia entre enero a diciembre de 2006, que firmaron el consentimiento informado y asistieron a todas las actividades programadas según el sílabo del Programa de Capacitación en Partograma con Curva de Alerta. Se excluyeron aquellos que no firmaron el consentimiento informado, no asistieron a las actividades programadas, o lo hicieron parcialmente.
La recolección de datos se realizó directamente por el investigador. Se hizo uso de las técnicas de la Encuesta y de la Observación. Para esto se elaboraron como Instrumentos un Cuestionario Individual con preguntas cerradas, con el cual se midió el nivel de conocimientos, y una Lista de Cotejo, para detectar la presencia o ausencia de determinadas características en la construcción del partograma por parte de los Internos de Medicina.

\section{CUADRO 1}

Indicadores utilizados para evaluar el nivel de aplicación en la elaboración del partograma por los internos de medicina.

\begin{tabular}{|c|c|}
\hline 1 & $\begin{array}{l}\text { Identifica claramente la hora cero para empezar a cons- } \\
\text { truir la curva de alerta. }\end{array}$ \\
\hline 2 & Identifica claramente la línea de base. \\
\hline 3 & $\begin{array}{l}\text { Registra datos de la paciente y la hora de las evaluaciones } \\
\text { en el espacio correspondiente. }\end{array}$ \\
\hline 4 & $\begin{array}{l}\text { En caso de parturientas con más de } 4-5 \mathrm{~cm} \text { de dilatación } \\
\text { al ingreso, hace coincidir la hora cero de la curva de alerta } \\
\text { con la hora cero de la curva real. }\end{array}$ \\
\hline 5 & $\begin{array}{l}\text { En caso de situaciones como cambio de posición de la pa- } \\
\text { ciente o rotura de membranas, traza una nueva curva de } \\
\text { alerta. }\end{array}$ \\
\hline 6 & $\begin{array}{l}\text { Conoce e identifica que en caso de realizar una nueva cur- } \\
\text { va de alerta, ésta debe continuar a la anterior desde la di- } \\
\text { latación cervical alcanzada en el momento de producirse } \\
\text { el cambio. }\end{array}$ \\
\hline 7 & $\begin{array}{l}\text { Registró el uso de oxitocina, fármacos o fluidos intrave- } \\
\text { nosos en el espacio correspondiente, si es que se usaron. }\end{array}$ \\
\hline 8 & Registró las evaluaciones en el espacio correspondiente. \\
\hline 9 & $\begin{array}{l}\text { Registró las características de las contracciones uterinas en } \\
\text { el espacio correspondiente. }\end{array}$ \\
\hline 10 & $\begin{array}{l}\text { En caso de situaciones de riesgo para la madre o el feto, } \\
\text { actúa según los protocolos. }\end{array}$ \\
\hline
\end{tabular}

La escala para la evaluación de conocimientos sobre el parto fue de 0-11, se consideraron los siguientes intervalos; Bueno 9-11, Regular 6-8, Malo 0-5. La evaluación de conocimientos sobre partograma tuvo una escala de $0-9$, se consideraron los siguientes intervalos; bueno 7-9, regular 4-6, malo 0-3. Para la evaluación de la aplicación en la elaboración del partograma, se consideraron 10 indicadores (Cuadro 1), se asignó 2 puntos por cada indicador cumplido y 0 si no se cumplía. De esta manera se construyeron los siguientes rangos; Muy bueno 17-20, bueno 13-16, regular 9-12, malo 5-8, muy malo 0-4. Para evaluar la confiabilidad del instrumento se utilizó el coeficiente alfa de cronbach $(\alpha=0.87)$.

La duración de la rotación del internado por el Departamento de Ginecología y Obstetricia es de tres meses. Durante 
la primera semana $\left(\mathrm{t}_{0}\right)$ de rotación se aplicó el cuestionario y la lista de cotejo, con el fin de obtener resultados sobre los conocimientos previos con que iniciaban la rotación. Durante la segunda y tercera semanas de rotación se realizó el programa de capacitación. Se elaboró un programa con un enfoque del proceso enseñanza de tipo constructivista, dando énfasis al alumno como centro del proceso y asumiendo el investigador un rol de facilitador. Se aplicó el modelo de la problematización, teniendo el Interno una participación activa. Se hizo uso de herramientas didácticas tales como exposiciones dialogadas, presentación de casos clínicos, observación de situaciones clínicas, demostraciones. Se usaron maquetas. El programa se desarrolló bajo la modalidad presencial. Además se brindó tutoría a aquellos Internos que se detectaron que no lograban los avances de acuerdo al programa. Esa tutoría no se esperó a que sea solicitada por el Interno, sino que fue dirigida según se detectaba el caso mediante la evaluación continua. A mitad de la rotación $\left(t_{1}\right)$, y al final $\left(t_{2}\right)$ de la misma se volvieron a aplicar el cuestionario y la lista de cotejo.

Los datos obtenidos se organizaron en una base de datos en el programa SPSS 15.0. Para el análisis estadístico univariado se tomó en cuenta la universidad de procedencia, la sede docente donde se realizó el curso de Obstetricia, el sexo y la edad. Para el análisis inferencial, se empleó las pruebas t de student para muestras independientes y el Análisis de Varianza ANOVA de una vía previa demostración de la homogenicidad de varianzas. Como análisis a posteriori (post hoc) se empleó el Test de de Scheffe. Los cálculos fueron realizados con un nivel confianza de $95 \%$.

\section{RESULTADOS}

\section{Características Generales}

Un total de 72 internos de medicina recibieron el Programa de Capacitación del Partograma con Curva de Alerta. 37.5\% fueron hombres y $62.5 \%$ mujeres; la edad promedio fue de 24.90 \pm 2.53 años con un rango de 22 a 35 años; y el grupo etáreo predominante fue el situado entre los 20 a 24 años (51.4\%).

Según las sedes de procedencia $70.8 \%$ realizó el curso de Obstetricia en una sede del Ministerio de Salud, $22.2 \%$ en EsSalud (Seguro Social del Perú) y 6.9 \% en las Fuerzas Armadas. En cuanto a la universidad de procedencia, $26.4 \%$ procedía de alguna universidad nacional y $73.6 \%$ de una universidad particular.

\section{NIVEL DE CONOCIMIENTOS DEL PARTO}

En el tiempo $t_{0}$ el nivel de conocimientos del parto fue predominantemente regular (38 internos), lo cual mejoró en el tiempo $t_{1}$ donde la calificación fue regular o buena y en el tiempo $t_{2}$ donde 40 internos alcanzaron el nivel de conocimientos bueno.
$\mathrm{Al}$ evaluarse el nivel de conocimientos sobre el parto en función del puntaje obtenido en los cuestionarios implementados al inicio de la rotación o basal $\left(\mathrm{t}_{0}\right)$, al final de la séptima semana de rotación $\left(\mathrm{t}_{1}\right)$ cuando han concluido las fases de capacitación y tutoría en la práctica clínica y al final de rotación en la décimo tercera semana $\left(t_{2}\right)$ se encontró diferencia estadísticamente significativa $(\mathrm{p}<0.001)$ con un aumento significativo de los puntajes en cada estación en comparación con la inmediata anterior. La sede hospitalaria $(p=0.485)$ así como el tipo de universidad de procedencia nacional o privada $(\mathrm{p}=0.422)$ no influyeron en el puntaje basal de los internos en el $\mathrm{t}_{0}$. (Gráfico 1)

\section{NIVEL DE CONOCIMIENTOS DEL PARTOGRAMA}

El segundo parámetro evaluado fue el nivel de conocimientos sobre el partograma. En el tiempo $t_{0}$ fue predominantemente malo (44 internos) mejorando en el tiempo $t_{1}$ donde la calificación fue regular y en el tiempo $t_{2}$ donde fue predominantemente regular (56/72) o bueno (12/72).

En función al puntaje obtenido en los cuestionarios aplicados en los tiempos $\left(t_{0}\right),\left(t_{1}\right)$ y $\left(t_{2}\right)$, se encontró diferencia estadísticamente significativa ( $p<0.001)$, (Gráfico 2$)$. La sede hospitalaria ( $p=0.598$ ) así como el tipo de universidad de procedencia $(p=0.773)$ no influyeron en el puntaje basal de los internos en el $t_{0}$.

\section{NIVEL DE APLICACIÓN EN LA ELABORACIÓN DEL PARTOGRAMA}

El nivel de aplicación en la elaboración del partograma en el tiempo $t_{0}$ fue predominantemente malo o muy malo (50 internos), mejoró en el tiempo $t_{1}$ donde 57 internos obtuvieron calificación de bueno o muy bueno y en el tiempo $t_{2}$ donde 71 alcanzaron la calificación de bueno o muy bueno.

Finalmente se evaluó el nivel de aplicación en la elaboración del partograma en función del puntaje obtenido en las listas de cotejo aplicadas en los tiempos $t_{0}, t_{1}$ y $t_{2}$, encontrándose una diferencia estadísticamente significativa $(p<0.001)$. (Gráfico 3). La sede hospitalaria $(p=0.101)$ así como el tipo de universidad de procedencia $(p=0.550)$ no influyeron en el puntaje basal de los internos en el $t_{0}$.

\section{DISCUSIÓN}

El estudio demuestra que el Programa de Capacitación de Partograma con Curva de Alerta cambia el nivel de conocimientos sobre parto y partograma, así como el nivel de aplicación para la elaboración del partograma por parte de los Internos de Medicina.

La utilización de programas de intervención para la mejora del rendimiento de los futuros médicos se ha realizado en 


\section{GrÁFICO 1}

Puntajes alcanzados en la evaluación del nivel de conocimientos del parto (NCP) por los internos de medicina en los tiempos $t_{0}, t_{1} \mathrm{y} \mathrm{t}_{2}$

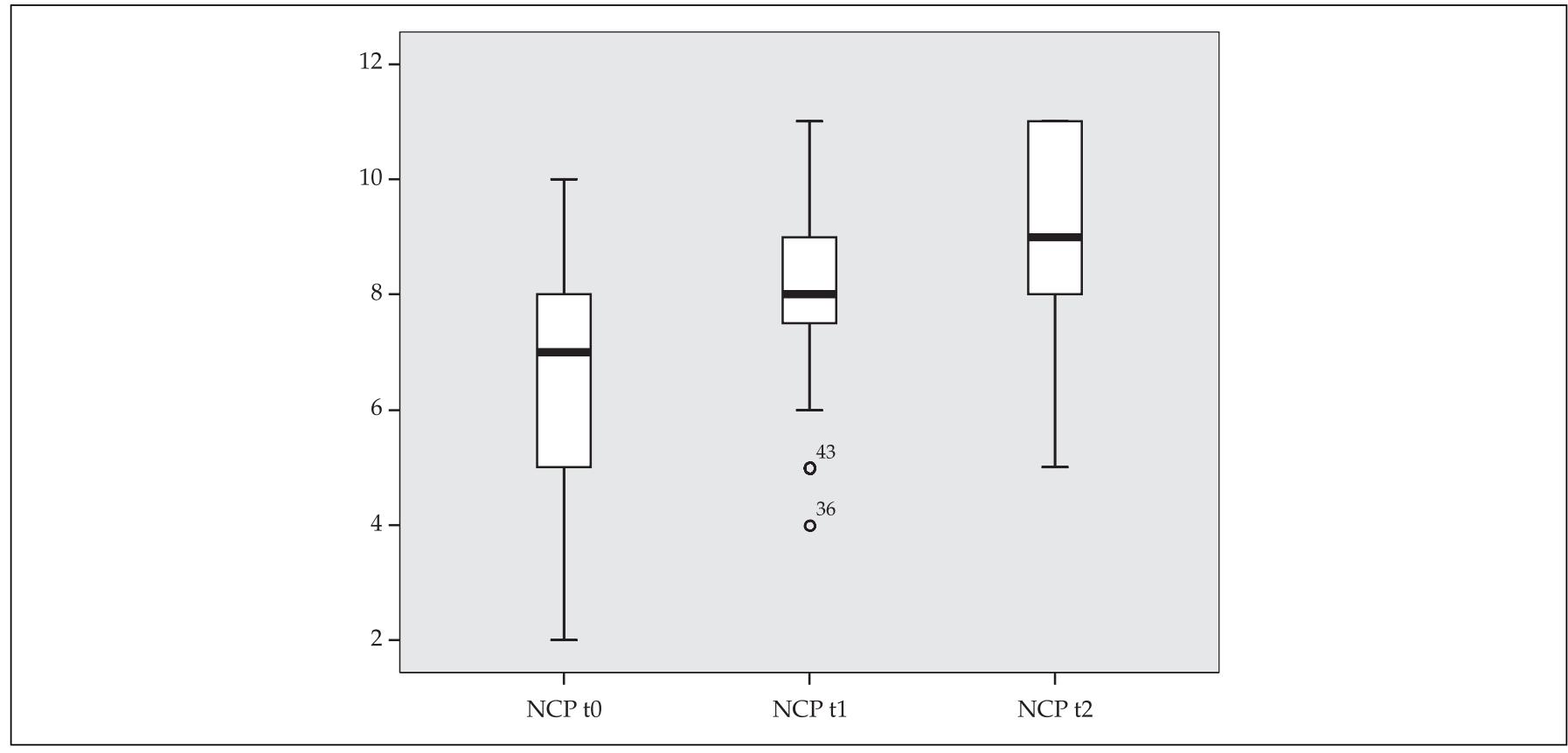

(*) Basado en un puntaje máximo de 11 puntos.

GRÁFICO 2

Puntajes alcanzados en la evaluación del nivel de conocimientos del partograma (NCPA) por los internos de medicina en los tiempos $t_{0}, t_{1} \mathrm{yt}_{2}$

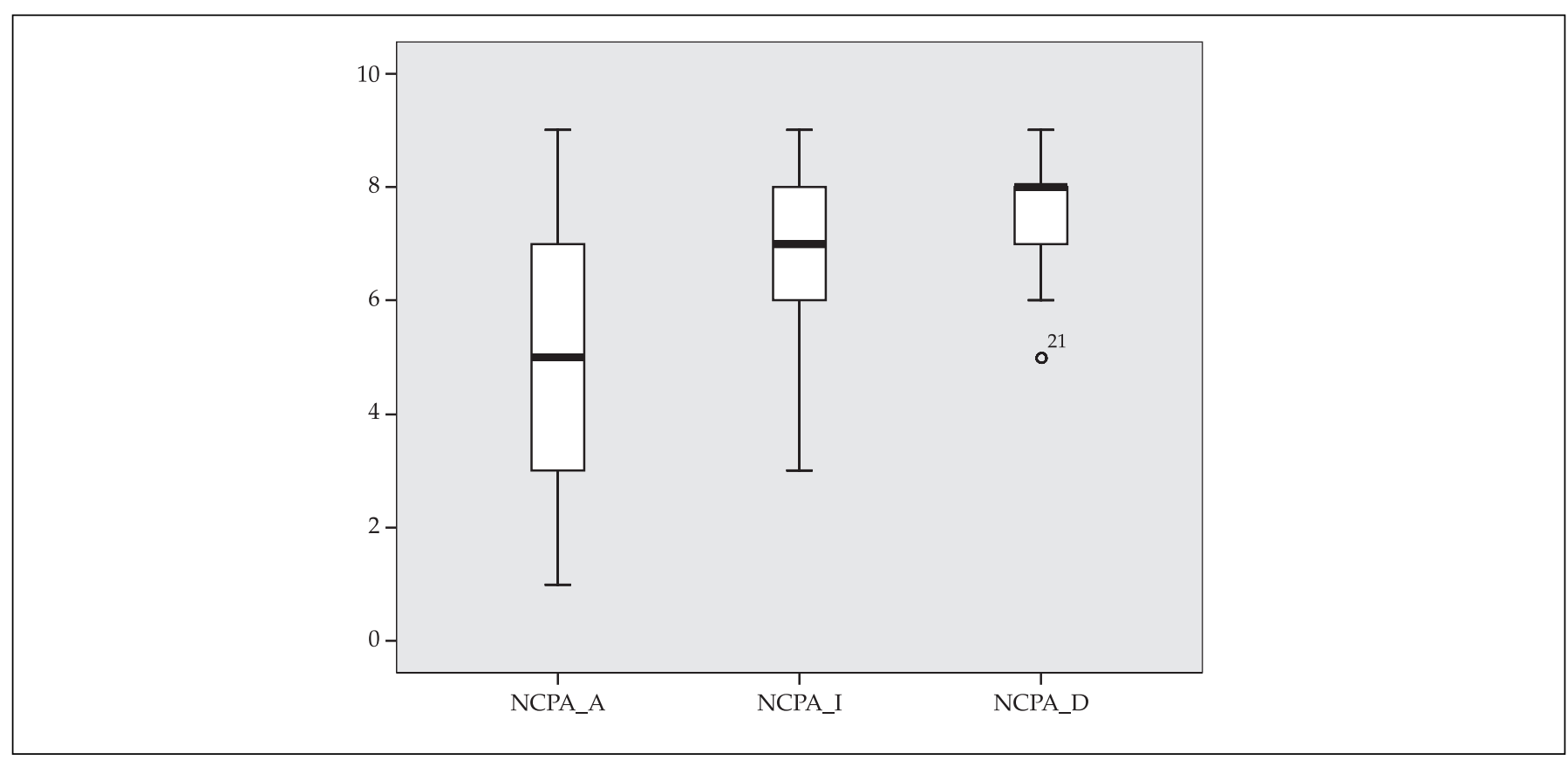

(*) Basado en un puntaje máximo de 9 puntos. 


\section{GrÁFICO 3}

Puntajes alcanzados en la evaluación del nivel de aplicación en la elaboración del partograma (NAPA) por los internos de medicina en los tiempos $t_{0}, t_{1} \mathrm{yt}_{2}$

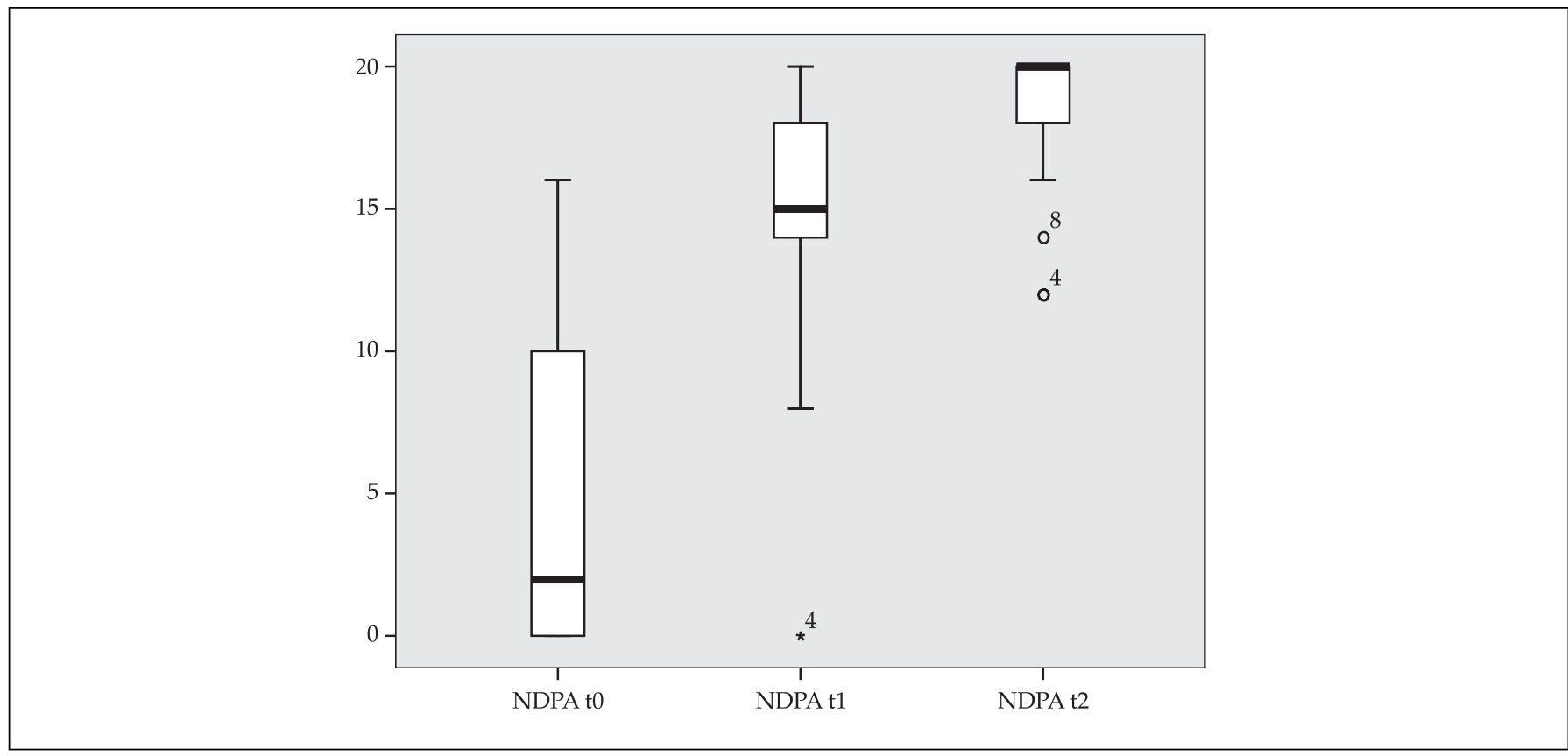

(*) Basado en un puntaje máximo de 20 puntos.

otras realidades, de esta manera un estudio realizado en USA demuestra que un programa acelerado de una semana antes del inicio del internado medico mejora el desempeño de los internos ${ }^{5}$.

Si bien los 72 internos que intervinieron en el estudio procedían de diferentes Facultades de Medicina a nivel nacional, y a su vez realizaron el curso de Obstetricia en diferentes sedes hospitalarias, esto no tuvo diferencia estadísticamente significativa en los puntajes basales que obtuvieron, cuando se exploraron los conocimientos previos con que contaban antes de recibir el programa de capacitación. Es importante remarcar esto, ya que en los sílabos de las diferentes facultades no se brinda igual importancia a la enseñanza de este instrumento.

Sin embargo, los puntajes basales $\left(\mathrm{t}_{0}\right)$ para los conocimientos sobre partograma y para la aplicación en la elaboración del partograma fueron malo y muy malo respectivamente. Lo cual confirma lo detectado en la práctica clínica rutinaria de esta falencia con la cual inician sus actividades los Internos de Medicina, siendo importante que los médicos egresados tengan el dominio del partograma, cualquiera sea su tipo, ya que son ellos los que controlan y asisten partos de bajo riesgo obstétrico, sin saber en qué momento se puede presentar una complicación del trabajo de parto, la cual debe ser diagnosticada y tratada oportunamente.

Además, en los diferentes hospitales a nivel nacional no se emplea el mismo tipo de partograma, pudiendo establecerse un sesgo en aquellos internos que procedían de sedes hospitalarias en las cuales no se utiliza el Partograma con Curva de Alerta, el cual sí es utilizado en el Hospital Nacional “Dos de Mayo", lo cual debería reflejarse más en la aplicación para la elaboración del mismo, y no tanto en los conocimientos sobre el partograma. Esto en razón a que todos los partogramas tienen muchas coincidencias en su base teórica, y más bien se diferencian en su diseño.

No ocurre lo mismo con el puntaje basal $\left(\mathrm{t}_{0}\right)$ para los conocimientos sobre parto, que fue regular; esto en probable relación a que lo referido a parto es de conocimiento universal, por lo que no se presentaría el sesgo mencionado para lo referente a conocimientos sobre partograma y su aplicación para la elaboración del mismo, ya que se supone que independientemente de la sede hospitalaria donde se realizó el curso los conocimientos adquiridos deben ser uniformes. 
En el estudio se aprecia que a través del tiempo, y habiendo recibido el programa de capacitación los internos de medicina progresaron en los puntajes obtenidos tanto para el nivel de conocimientos de parto y partograma, como para el nivel de aplicación en la elaboración del partograma. En este último, el progreso es de muy malo a muy bueno en la mayoría, es decir desde un nivel mínimo a uno máximo. Mientras que en el nivel de conocimientos sólo hay un progreso al nivel inmediato superior, de regular a bueno en conocimientos sobre parto, y de malo a regular en conocimientos sobre partograma. Se esperaría que los niveles de conocimientos sobre partograma hubieran llegado al límite superior, como ocurre con el nivel de aplicación para la elaboración del partograma, sin embargo un limitante a esto es la dificultad para medir conocimientos, lo cual hemos pretendido con la aplicación del cuestionario, y no así cuando se ha medido el nivel de aplicación para la elaboración del partograma ya que el interno combina los contenidos conceptuales y procedimentales de las competencias que ha adquirido.

Siguiendo el Modelo de los cuatro niveles de Kirkpatrick ${ }^{6}$, para evaluar las acciones formativas, se lograron los objetivos para el nivel II y el nivel III. En el nivel II o de Aprendizaje, la evaluación formativa y sumativa demostró el cambio tanto en conocimientos como en habilidades por parte de los internos luego de recibir el programa de capacitación. Además permitió una retroalimentación para mejorar y lograr las competencias esperadas. En el nivel III o de comportamiento, permitió a los internos de medicina conducir de una mejor manera los trabajos de parto asignados, así como la detección oportuna de complicaciones. En el estudio no fueron evaluados los niveles I, de reacción, ni IV, de resultados o impacto. El primero por no haber sido diseñado en el estudio, y el segundo porque este nivel de evaluación debería realizarse durante la práctica profesional, es decir como médicos titulados.

En el campo educativo, la mayoría de las facultades y escuelas de Medicina, asociaciones nacionales e internacionales y colegios de profesionales del continente están debatiendo sobre cómo educar mejor a los futuros médicos para dar respuesta al problema de salud actual; se critica fuertemente la rigidez del curriculum, la falta de integración en las materias, el carácter pasivo de la enseñanza, la ausencia del desempeño del rol de la universidad para cumplir con sus tres funciones básicas: la docencia, la investigación y la extensión, entre muchos otros temas ${ }^{7}$. Adicionalmente es importante recordar que muchas veces los estudiantes de pregrado consideran que sus cursos clínicos son de corta duración para satisfacer sus expectativas de aprendizaje ${ }^{8}$, e incluso muchos de ellos invierten buena parte de su tiempo libre reforzando sus conocimientos al lado de los pacientes ${ }^{9}$. Por lo tanto, recalcamos la importan- cia de realizar programas de capacitación a internos de medicina antes de iniciar sus labores.

En nuestro medio actualmente existe interés creciente en la educación médica contemporánea por el perfeccionamiento del proceso de enseñanza-aprendizaje, para elevar la calidad en los procesos de formación de los recursos humanos de la Salud y satisfacer las necesidades de la población ${ }^{10}$.

Sin embargo, el presente estudio siendo una muy pequeña parte de lo que se debe de enseñar en la carrera médica nos hace inferir que aunque se pretende una mayor independencia cognitiva del alumno, todavía las actividades en el aula tienen gran peso en su preparación, con predominio del método expositivo que conlleva a un aprendizaje pasivo y reproductivo. Esto indudablemente nos lleva a pensar que deben aplicarse métodos de enseñanza-aprendizaje que modifiquen la actitud de los participantes hacia una posición activa y productiva de autoestudio, autodisciplina y autocontrol, para lograr un pensamiento independiente y responsable, que los prepare para su actuación futura en el equipo de salud ${ }^{11}$.

\section{CONCLUSIONES}

El presente estudio con los resultados estadísticamente significativos en el cambio de los niveles de conocimiento luego de realizar la intervención mediante un programa de capacitación, demuestra el impacto logrado utilizando un diseño de pre y post test, como ocurre cuando se utiliza dicho diseño ${ }^{12}$. Pero, lo fundamental es el tipo de enfoque que se dio al proceso de enseñanza aprendizaje, el cual fue de tipo constructivista, donde coincidimos que la enseñanza tutelar logra una interacción entre el tutor y el alumno, haciendo que el aprendizaje se centre en este último ${ }^{13}$. Este enfoque del proceso enseñanza-aprendizaje debería ser una forma contemporánea de aplicar los principios didácticos de la enseñanza que establecen, entre otros, la vinculación de la teoría con la práctica, de lo concreto con lo abstracto y de lo individual con lo colectivo, así como el carácter consciente de la actividad del estudiante.

Menciones: basado en "Aparicio J. Programa de capacitación de partograma con curva de alerta y cambios en el nivel de conocimientos para su elaboración por los internos de medicina del hospital nacional Dos de Mayo. Enero - diciembre 2006. [Tesis para optar el grado de Magister en Docencia e Investigación en Salud]. Lima: Universidad Nacional Mayor de San Marcos; 2006".

\section{REFERENCIAS}

1. Luckie DB, Maleszewski JJ, Loznak SD, Krha M. Infusion of collaborative inquiry throughout a biology curriculum increases student learning: a four-year study of "Teams and Streams". Adv Physiol Educ. 2004;28:199-209. 
2. Venturelli J. Educación Médica: Nuevos enfoques, metas y métodos. Washington, D.C.: OPS; 2003.

3. Moreira AM, Souza da Silva SH. Conceições do processo ensino-aprendizagem: um estudo com professores de medicina. Rev Bras Educ Med 2009;33:624-32.

4. Mathai M. The partograph for the prevention of obstructed labor. Clin Obstet Gynecol. 2009; 52:256-69.

5. Laack TA, Newman JS, Goyal DG, Torsher LC. A 1-week simulated internship course helps prepare medical students for transition to residency. Simul Healthc. 2010;5:127-32.

6. Nolla-Domenjó M. La evaluación en educación médica. Principios básicos. Educ Med. 2009; 12:223-9.

7. Morales I, Borroto R, Fernández B. Políticas y estrategia para la transformación de la educación superior en América Latina y el Caribe. Educ Med Super. 2005;19(1).

8. Hilasaca R, Ramos W, Galarza C. Percepción y satisfacción de los estudiantes del cuarto año de la escuela de Medicina Humana de la UNMSM acerca de las prácticas clínicas de Dermatología. Noviembre 2008. Dermatol Perú. 2009;19:178-88.

9. Murray E, Alderman P, Coppola W, Grol R, Bouhuijs P, van der Vleuten $C$. What do students actually do on an internal medicine clerkship? A log diary study. Med Educ. 2001;35:1101-7.

10. Cañizares O, Sarasa N. Una propuesta didáctica ante los problemas cognoscitivos en Anatomía Humana. Educ Med Super. 2004;18(4).

11. Camejo D, Arocha C. Evaluación del programa de estudio del diplomado Gerencia en Salud. Educ Med Super. 2005;19(2).

12. Flores P, Diaz P. Evaluación de un programa de capacitación para el personal de Salud sobre atención y abordaje de adolescentes en La Paz, Bolivia. Educ Med Super. 2004;18(1).

13. Lugones M, García M, Pichs L. La enseñanza tutelar y los profesores principales en el proyecto del policlínico universitario. Educ Med Super. 2005;19(1).

\section{CONTRIBUCIÓN DE LOS AUTORES}

Jorge Renato Aparicio Ponce diseñó el estudio y realizó la recolección de datos, Gerardo Ronceros realizó la búsqueda bibliográfica y preparó el protocolo, Karim Goebel escribió el artículo. Ericson L. Gutierrez realizó el análisis estadístico. Todos los autores revisaron y aprobaron la versión final del artículo de investigación.

\section{CONFLICTO DE INTERESES}

Declaró que no hubo.

\section{CORRESPONDENCIA PARA EL AUTOR}

Dr. Jorge Renato Aparicio Ponce.

Calle Sor Tita, 281

Urbanización Benavides. Miraflores

Lima 18 - Perú.

E-Mail: jrapariciop@yahoo.es

\section{UM PROGRAMA DE TREINAMENTO PRÉVIO AO INÍCIO DA PRÁTICA PROFISSIONAL DE MÉDICOS PERUANOS MELHORA SEUS CONHECIMENTOS SOBRE O ATENDIMENTO AO PARTO E O PARTOGRAMA}

\section{RESUMO:}

Foi realizado um estudo analítico, longitudinal e prospectivo; foi aplicado um questionário e uma lista de verificação para determinar o nível de conhecimentos e aplicação prévios sobre o parto e o partograma a 72 internos de medicina que, em sistema de rodízio, passaram pelo serviço de Ginecologia e Obstetrícia do Hospital Nacional Dos de Mayo de Lima-Peru, entre janeiro e dezembro de 2006. Posteriormente, foi aplicado o mesmo instrumento na metade e no final do estudo. Para a análise estatística, foi utilizado o teste de análise de variância (ANOVA), os cálculos foram realizados com um nível de confiança de 95\%. O resultado apresentou um aumento significativo no nível de conhecimentos sobre o parto, o partograma e a aplicação na elaboração do partograma $(p<0.001)$. A importância do estudo reside no fato que a maioria dos médicos peruanos vai iniciar a sua prática profissional em serviços de atendimento médico primário longe dos centros médicos de maior complexidade, nos quais eles vão ter que realizar partos. Além disso, este programa vai ajudar eles a reconhecer o momento indicado para realizar una transferência para um centro de saúde de maior complexidade.

\section{PALAVRAS-CHAVE:}

$$
\begin{aligned}
& \text { - Partograma. } \\
& \text { - Parto. } \\
& \text { - Educação. }
\end{aligned}
$$

